\title{
Grip force control during object manipulation in cervical myelopathy
}

\author{
Naoto Noguchi ${ }^{1} \cdot$ Bumsuk Lee $^{2} \cdot$ Shoya Kamiya ${ }^{3} \cdot K$ Ken Kondo ${ }^{4} \cdot$ Ryoto Teshima $^{5} \cdot$ Masatake Ino $^{6} \cdot$ Koji Tanaka $^{2} \cdot$ \\ Tsuneo Yamazaki ${ }^{2}$
}

Received: 26 September 2019 / Revised: 3 January 2020 / Accepted: 6 January 2020 / Published online: 15 January 2020

(c) The Author(s), under exclusive licence to International Spinal Cord Society 2020

\begin{abstract}
Study design Cross-sectional research.

Objectives To objectively evaluate grip force (GF) control while holding a freely movable object in individuals with cervical myelopathy (CM).

Setting Harunaso Hospital, Takasaki, Japan.

Methods We studied 52 hands from 26 individuals with CM. Participants performed a grip-and-lift task by pulp pinch using the thumb and index finger before surgery. We monitored individual finger GF (N) during the first $3 \mathrm{~s}$ while lifting and holding an object. Correlations between the GF and other clinical tests were evaluated. A multiple stepwise regression analysis was used to examine the contribution of the GF to the severity of clinical symptoms.

Results Thumb GF was negatively correlated with the 10-s test (rs = -0.32), and index finger GF was positively correlated with its cutaneous pressure threshold ( $\mathrm{rs}=0.34$ ). Multiple regression for the severity of upper extremity symptoms revealed that the model including the GF had a larger adjusted $R^{2}$ and a lower AIC value than that of conventionally used clinical tests.

Conclusions These results suggested that the assessment of individual finger GF control could provide an indicator of the clinical severity of upper extremity in individuals with CM.
\end{abstract}

\section{Introduction}

Cervical myelopathy (CM) is a condition caused by spinal cord compression resulting from unspecified degenerative changes and segmental instability $[1,2]$. Individuals with CM generally suffer from motor and sensory symptoms in

Bumsuk Lee

leebumsuk@gunma-u.ac.jp

1 Faculty of Rehabilitation, Gunma University of Health and Welfare, Maebashi, Japan

2 Gunma University Graduate School of Health Sciences, Maebashi, Japan

3 Department of Rehabilitation, Harunaso Hospital, Takasaki, Japan

4 Department of Rehabilitation, Fujioka General Hospital, Fujioka, Japan

5 Division of Rehabilitation Service, Geriatrics Research Institute and Hospital, Maebashi, Japan

6 Gunma Spine Center, Harunaso Hospital, Takasaki, Japan the four limbs, such as clumsiness and paresthesia [3, 4]. Hand clumsiness is one of the most common complaints in CM [5]. This hand dysfunction, also called myelopathy hand, is characterized by motor weakness, exaggerated deep tendon reflexes, loss of sensitivity, and difficulty in rapid grip-and-release movement of the fingers [6, 7]. These symptoms affect the functional independence of upper limb performance in activities of daily living (ADL), such as eating, dressing, and writing.

Several scales have been developed to assess clinical deficits in individuals with CM. The Japanese Orthopedic Association (JOA) score is one of the most frequently used assessments, and focuses on neurological function of the four extremities [8]. In addition to the JOA score, the 10-s test is a simple test for evaluating hand disability. It is known that patients with severe CM could only complete approximately eight times, although able-bodied participants will complete more than 20 cycles in $10 \mathrm{~s}$ [9]. However, movements in the 10 -s test are quite different from the upper limb movements in ADL, because the test focuses on total finger flexion/ extension rather than individual finger movement. 
Grip force (GF) control while holding an object is a fundamental assessment of hand dysfunction. Exaggerated GF is considered to be a strategic response to sensory deficits [10] and decreased muscle activity caused by damage to the corticospinal tract [11]. Akutagawa et al. suggested that individuals with severe $\mathrm{CM}$ also tend to use a greater GF by the three-point pinch by their thumb, index, and middle finger to avoid dropping objects, compared with moderate $\mathrm{CM}$ and able-bodied participants [9]. It is suggested that exaggerated GF implies altered sensorimotor control of grasping.

Evaluation of GF control, therefore, is necessary to quantitatively and objectively measure hand dysfunction. However, in contrast to numerous GF studies on stroke groups [10, 12,13], the characteristics of GF control in CM and its relationship with clinical tests (e.g., sensation, muscle strength, and hand dexterity) have not yet been adequately discussed. The aim of this study was to evaluate the kinetic characteristics of individual finger GF in CM using a precision grip-and-lift task. We also investigated the relationship between GF trace and other clinical tests.

\section{Methods}

\section{Participants}

We studied 52 hands from 26 individuals with CM (18 males and 8 females, $66.3 \pm 12.6$ years). The mean body mass index was $24.2 \pm 2.8 \mathrm{~kg} / \mathrm{m}^{2}$. Participants were diagnosed as having cervical spondylotic myelopathy (23 participants) or ossification of the posterior longitudinal ligament (3 participants), and all participants were waiting for surgical operations. The median duration from symptom onset was 12 months (IQR 4 to 60). Magnetic resonance imaging was performed for all participants, and the results showed cervical cord stenosis. Maximal cord compression were at $\mathrm{C} 3 / 4$ in eleven patients, at $\mathrm{C} 4 / 5$ in seven, at C5/6 in seven, and $\mathrm{C} 6 / 7$ in one. Based on the impairment scale developed by the American Spinal Injury Association, all individuals were in D scale; with motor function preserved below the neurological level, and more than half of the key muscles were of a grade greater than three. They were able to walk with or without walking aid. The inclusion criteria were (1) clinical diagnosis of CM and no history of cervical spine surgery, (2) ability to perform the holding task with the thumb and index finger, and (3) ability to follow commands and having no visual disturbances or cognitive deficits. The exclusion criteria were (1) history of previous cervical spine surgery, and (2) other disorders that might impair grip-and-lift performance. Informed consent was obtained from all participants prior to the study. To evaluate the deficit of GF control in individuals with CM, data on 42 hands from 21 able-bodied controls ( 3 males and 18 females, $22.0 \pm 2.2$ years) were also collected. The study was approved by the University Ethical Review Board for Medical Research Involving Human Subjects.

\section{Grip-and-lift task}

The experimental procedure was performed in accordance with Westling and Johansson [14]. The participants sat on a chair facing a height-adjustable table, and the finger pads were wiped with alcohol swabs to reduce inter-individual variability in finger skin friction. An iron cube (weight: $250 \mathrm{~g}, 31 \times 31 \times 31 \mathrm{~mm}$ ) was placed on the support height of $7.5 \mathrm{~cm}$, and $30 \mathrm{~cm}$ from the participants in the midsagittal plane. Verbal instructions were given to grip the cube by the pulp pinch using the thumb and index finger, lift it $\sim 5 \mathrm{~cm}$, and hold for $\sim 5 \mathrm{~s}$ (Fig. 1). The participants were also instructed to use minimal force to perform the task. Before each lift, participants were allowed to touch the cube with minimal GF. The lifting tasks were repeated ten times for each hand. A 5-s rest was taken between each lift, and a 5 -min rest was provided between right and left hands. The testing order for right and left hands was randomized.

\section{Materials}

Two pressure sensor sheets (Pressure Mapping Sensor 5027, Tekscan, South Boston, MA, USA) were attached to the grip surfaces of the cube. Each sheet had a sensing area of 27.9 by $27.9 \mathrm{~mm}$ with 1936 sensing elements (sensels) distributed over 44 rows and 44 columns, a thickness of $0.1 \mathrm{~mm}$, and a sensitivity range of $0-345 \mathrm{kPa}$ for each sensel. Before each recording, the sensor sheet was equilibrated and calibrated using the test instrument. The pressure disturbance was recorded to detect the magnitude of GF by

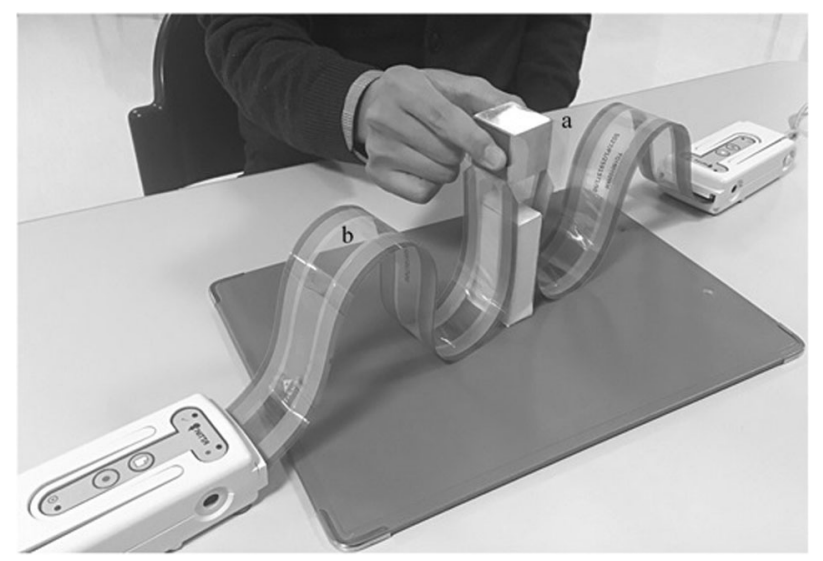

Fig. 1 The grip-and-lift task. Participants gripped and lifted an iron cube $(\mathbf{a} 250 \mathrm{~g})$ using the thumb and index finger pulps. The iron cube was placed on the support height of $7.5 \mathrm{~cm}$, and two pressure sensor sheets (b) were attached to the grip surfaces of the cube. 
using the I-Scan 100 System (Nitta, Japan) at a frequency of $100 \mathrm{~Hz}$ in 8-bit resolution. For each lift, the GF was calculated from the sum of the pressure in all 1936 sensels during the first $3 \mathrm{~s}$ since the force of $0.5 \mathrm{~N}$ was initially loaded on a sensel.

\section{Clinical tests}

Other clinical tests were performed in random order separately from the grip-and-lift task 1-9 days prior to the surgery. The severity of physical disability was assessed using the latest version of the JOA score in Japanese [8]. This scale consists of six domains scores: motor dysfunction in the upper extremities, motor dysfunction in the lower extremities, sensory function in the upper extremities, sensory function in the trunk, sensory function in the lower extremities, and bladder function. The minimum total score is 0 and the maximum is 17. Although the JOA score includes functions of the lower extremity and bladder, some studies have employed pertinent sub scores of upper extremity function from the JOA score in isolation [9, 15]. In accordance with these studies, we selected the sub score related to finger motor function from the JOA score, and defined it as JOA-UEF for the purpose of the present study. The JOA-UEF is assessed from 0 to 4 points: $0=$ unable to feed oneself with any tableware, including chopsticks, a spoon, or fork, and/or unable to fasten buttons of any size; $1=$ can manage to feed oneself with a spoon and/or a fork but not with chopsticks; 2 = either chopstick feeding or writing is possible but not practical, and/or large buttons can be fastened; $3=$ either chopstick feeding or writing is clumsy but practical, and/or cuff buttons can be fastened; $4=$ normal.

The maximum grip strength was measured with the Smedley Grip Tester (Gopher, Owatonna, MN, USA), and pinch strength between thumb and index finger was measured with the JAMAR Hydraulic Pinch Gauge (Petterson Medical, Warrenville, IL, USA). The cutaneous pressure threshold of the central areas of the thumb and index finger pulps was assessed with Touch Test Sensor Evaluators (North Coast Medical Inc., Morgan Hill, CA, USA), which apply standard calibrated forces of $0.07,0.4,2.0,40$, and $300 \mathrm{~g}$, respectively. The lowest perceived monofilament is considered normal, and higher monofilament forces indicate the severity of sensory disturbance. In the 10-s test, individuals were asked to perform full grip-and-release with the hands as fast as possible, for $10 \mathrm{~s}$. Dexterity function was also assessed by three subtests (numbers 8, 9, and 10) of the Simple Test for Evaluating Hand Function (STEF) [16]. These three subtests required participants to manipulate various small objects. For example, in subtest 8 , participants reach and pinch a circular disk $(3 \mathrm{~cm}$ in diameter and $1 \mathrm{~cm}$ thick) and then bring the disk into a target space as quickly as possible, repeating this process six times. The time to complete each subtest was used for statistical analysis.

\section{Statistical analysis}

This study was cross-sectional study to investigate whether GF control provide an indicator of the clinical severity of upper extremity in individuals with CM.

Difference between the CM and the control groups was examined using Mann-Whitney test. Associations between individual finger GF and other clinical tests were examined with Spearman's rank correlation. To determine which variable best predicted the severity of clinical symptoms, a stepwise multiple regression analysis was performed with the JOA-UEF as the dependent variable, and individual GF, grip strength, pinch strength, the 10-s test, and time of STEF as the explanatory variables. Goodness of fit was determined using adjusted $R^{2}$ and the Akaike information criteria (AIC). The AIC provides a way to relatively compare models that have different input variables. The preferred model is that with the lowest AIC value. The statistical software SPSS ver. 25.0 J for Windows (SPSS Japan, Tokyo, Japan) was used for the analysis. Values of $p<0.01$ were considered significant.

\section{Results}

The mean JOA score in individuals with $\mathrm{CM}$ was $11.1 \pm 2.3$ points, and the JOA-UEF was $2.5 \pm 0.8$ points

Table 1 shows the results of comparison between the CM and the control groups. In individuals with CM had significantly larger GFs than control group, although grip/ pinch strengths were not significantly different.

The results of correlations between the GF and upper extremity functional tests in individuals with $\mathrm{CM}$ are shown in Table 2. The GFs were significantly correlated with the JOA-UEF and time of STEF. Moreover, the 10-s test was negatively correlated with thumb GF, and cutaneous threshold pressure of the index finger was positively associated with the thumb and index finger GF.

To further investigate whether the GF could explain the clinical symptoms in addition to the conventional clinical tests, a stepwise multiple regression analysis was performed with two models (Table 3). Model 1 with conventional clinical tests showed that the JOA-UEF could be predicted by the time of STEF $(\beta=-0.56, p<0.01)$; the adjusted $R^{2}$ value was 0.30 and the AIC value was -46.76 . Model 2 , in which finger GFs were included in addition to the independent variables used in Model 1, revealed that the time of STEF $(\beta=-0.42, p<0.01)$ and thumb GF $(\beta=-0.35, p<$ $0.01)$ were significantly associated with the JOA-UEF; the adjusted $R^{2}$ was 0.39 and AIC value was -52.89 . 


\section{Discussion}

We found that deficit of GF control was associated with the severity of hand dysfunction in CM. Moreover, it was found that the thumb GF was correlated with the 10-s test, and index finger GF was correlated with its cutaneous pressure threshold.

The multiple regression model for predicting JOA-UEF incorporating the grip-and-lift task had a larger adjusted $R^{2}$ and the lower AIC value compared with only the commonly used clinical tests (Table 3). Moreover, we also found that

Table 1 Summarized data from precision grip-and-lift task and conventional clinical tests.

\begin{tabular}{lccc}
\hline & $\begin{array}{l}\text { CM group } \\
(n=52) \\
\text { Mean (SD) }\end{array}$ & $\begin{array}{l}\text { Control group } \\
(n=42) \\
\text { Mean (SD) }\end{array}$ & $P$ value \\
\hline Grip force during the first 3 s (N) & & \\
$\quad$ Thumb & $1367.1(979.3)$ & $728.3(360.6)$ & $<0.01$ \\
$\quad$ Index finger & $1498.7(1033.6)$ & $772.6(375.7)$ & $<0.01$ \\
Grip strength (kg) & $25.9(9.6)$ & $28.5(8.0)$ & 0.11 \\
Pinch strength (kg) & $3.9(1.8)$ & $3.3(0.7)$ & 0.19 \\
10-s test (number) & $16.0(4.2)$ & - & - \\
Cutaneous pressure threshold (g) & & $<0.01$ \\
$\quad$ Thumb & $2.2(7.8)$ & $0.1(0.0)$ & $<0.01$ \\
$\quad$ Index finger & $1.2(5.6)$ & $0.1(0.0)$ & - \\
STEF (s) & $42.5(12.3)$ & - &
\end{tabular}

Mann-Whitney test

STEF Simple Test for Evaluating Hand Function
GF was associated with the JOA-UEF, which focuses on the severity of upper limb movements in ADL. This grip-andlift task is a sensitive measure for assessing grasping disability in individuals with central nervous system disease. For example, the GF of the paretic hand is significantly increased compared with the nonparetic hand in stroke [17]. An unnecessarily high GF was used to avoid dropping objects in individuals with $\mathrm{CM}$ compared to able-bodied controls [9]. In our previous study, GF was significantly correlated with STEF subtest times [18]. Our results are consistent with these previous studies. It is thought that compression of the spinal cord leads to impairments of GF control, and affects upper extremity performance. Therefore, it is reasonable to consider the deficit of GF control as one of the clinical symptoms of CM.

Although sensory disturbance is also a clinical symptom of CM [19, 20], it was not significantly associated with the JOAUEF. This finding may be explained by the methodology of JOA-UEF. The score is based on assessments of upper extremity performance in daily living, such as eating with chopsticks or a spoon and dressing $[9,15]$. The participants had no visual problems, and therefore could perform these movements using visual compensation. Akutagawa et al. reported that people who suffer from CM apparently failed to maintain GF with their eyes closed [9]. Future studies should consider the performance of daily actions without visual compensation (e.g., taking coins out from a wallet).

Another key finding of the study was correlations between kinetic parameter and clinical test. We found that a negative correlation between the 10-s test and thumb GF (Table 2). Hand dysfunction is well-represented by thumb

Table 2 Correlations between the GF and upper extremity functional tests in individuals with cervical myelopathy.

\begin{tabular}{|c|c|c|c|c|c|c|c|c|c|c|c|c|c|c|}
\hline & \multirow{2}{*}{\multicolumn{2}{|c|}{ JOA-UEF }} & \multirow{2}{*}{\multicolumn{2}{|c|}{ Grip strength }} & \multirow{2}{*}{\multicolumn{2}{|c|}{ Pinch strength }} & \multirow{2}{*}{\multicolumn{2}{|c|}{10 -s test }} & \multicolumn{4}{|c|}{ Cutaneous threshold } & \multirow{2}{*}{\multicolumn{2}{|c|}{ STEF }} \\
\hline & & & & & & & & & \multicolumn{2}{|c|}{ Thumb } & \multicolumn{2}{|c|}{ Index finger } & & \\
\hline & rs & $P$ value & rs & $P$ value & rs & $P$ value & rs & $P$ value & rs & $P$ value & rs & $P$ value & rs & $P$ value \\
\hline \multicolumn{15}{|l|}{ Grip force } \\
\hline Thumb & -0.44 & $<0.01$ & -0.22 & 0.12 & -0.21 & 0.13 & -0.32 & 0.02 & 0.24 & 0.09 & 0.37 & $<0.01$ & 0.46 & $<0.01$ \\
\hline Index finger & -0.39 & $<0.01$ & -0.12 & 0.39 & -0.11 & 0.44 & -0.23 & 0.10 & 0.17 & 0.18 & 0.34 & 0.01 & 0.36 & 0.01 \\
\hline
\end{tabular}

JOA Japanese Orthopedic Association, UEF upper extremity function, STEF Simple Test for Evaluating Hand Function

Table 3 Results of multiple regression analysis for predicting JOA-UEF score.

\begin{tabular}{|c|c|c|c|c|c|c|}
\hline & $\begin{array}{l}\text { Dependent } \\
\text { variable }\end{array}$ & Adjusted $R^{2}$ & AIC value & $\begin{array}{l}\text { Explanatory } \\
\text { variable }\end{array}$ & Standardized $\beta$ & $P$ value \\
\hline Model 1: conventional clinical tests & JOA-UEF & 0.30 & -46.76 & STEF & -0.56 & $<0.01$ \\
\hline \multirow{2}{*}{$\begin{array}{l}\text { Model 2: conventional clinical tests + grip- } \\
\text { and-lift task }\end{array}$} & JOA-UEF & 0.39 & -52.89 & STEF & -0.42 & $<0.01$ \\
\hline & & & & Thumb GF & -0.35 & $<0.01$ \\
\hline
\end{tabular}

JOA Japanese Orthopedic Association, UEF upper extremity function, AIC Akaike information criteria, STEF Simple Test for Evaluating Hand Function, $G F$ grip force 
motor impairment, because the thumb position plays a key role in hand function [21]. Animal experiments in monkeys following spinal pyramidotomy showed that the thumb movement was not sufficient while manipulating objects [22]. Also, the force direction of the paretic thumb shows more deviation than the index finger [17, 23]. In CM, longer conduction times are reported in the abductor pollicis brevis $[4,24]$. These results suggested greater presence of corticospinal projections to the thumb muscles than to the index finger.

Index finger sensory function was found to be significantly correlated with finger GFs (Table 2). This result is consistent with a previous study that reported the index finger sensory function correlates with finger muscle activity during precision gripping in individuals with spinal cord injuries [25]. These observations may suggest that tactile afferent information in fingers, especially index finger may contribute to the GF control. Evaluating separately individual finger function is, therefore, necessary for improved understanding of hand dysfunction in CM.

Several limitations of the present study should be acknowledged. Firstly, the individuals' recovery over the long term was not monitored. To analyze the recovery process of precision grip function, a future study monitoring postoperative changes should be considered. Secondly, the tactile feature of the sensor sheet and contribution of vision were not considered in this study. It was known that the GFs varied with surface structure including surface roughness and the friction [26], and visual information [9]. It was possible that these conditions could influence the sensorimotor control of grasping. Thirdly, we could not exclude learning effect over ten trials while gripping and lifting the cube. Fourthly, we did not monitor wrist movements using a motion capturing system during the task. It was known that individuals with $\mathrm{CM}$ tend to compensate for the impaired finger motions with paradoxical wrist motion [7]. Therefore, the relationships between symptom of the wrist movements and GF remain unclear. Finally, we used JOA-UEF to assess the severity of clinical upper extremity symptom. It was reported that the sensitivity to change of the JOA score was likely to poor, because this scale classifies wide range of clinical severity using only a few arbitrary units [27]. In the future, we need to use more sensitive and comprehensive upper extremity function assessment tools such as the Graded Redefined Assessment of Strength, Sensation and Prehension [28].

This study demonstrated that the model including GF control had the larger adjusted $R^{2}$ and the lower AIC than that of conventionally used clinical tests, suggesting that the GF during grip-and-lift task was useful for assessing in individuals with $\mathrm{CM}$. In terms of the motor examination, in the upper extremities, muscle strength has traditionally been widely used in clinical settings. Although the muscle force output is a good clinical parameter to measure the level of upper extremity weakness, it does not provide the information on functional use of the upper extremity. Compared with that, the GF control during grip-and-lift task provides the information on the ability to modulate muscle activity, indicating fundamental basis of hand function. Thus, the ability to grip in individuals with CM may be an important indicator of the functional use of the upper extremity. Based on the study, we conclude that the assessment of individual finger GF control could provide an indicator of the clinical severity of upper extremity in individuals with CM. This new knowledge may improve our understanding of upper limb performance for early diagnosis and rehabilitation progress in $\mathrm{CM}$.

\section{Data availability}

The datasets generated and analyzed during the current study are available from the corresponding author on reasonable request.

Acknowledgements We would like to thank Haruyasu Fujita (Gunma University, Department of Public Health, Maebashi, Japan) for his valuable statistical advice during the preparation of this paper.

Author contributions NN, BL, and TY conceptualized and designed the study. NN, SK, and MI prepared and coordinated data analyses and interpretations. NN, KK, and RT analyzed the data. KT provided statistical support in addition to critical feedback of the paper. All authors participated in the critical review and afterward approved the paper.

\section{Compliance with ethical standards}

Conflict of interest The authors declare that they have no conflict of interest.

Ethical approval We certify that all applicable institutional and governmental regulations concerning the ethical use of human volunteers were followed during the course of this research.

Publisher's note Springer Nature remains neutral with regard to jurisdictional claims in published maps and institutional affiliations.

\section{References}

1. Liang ZH, Di Z, Jiang S, Xu SJ, Zhu XP, Fu WB, et al. The optimized acupuncture treatment for neck pain caused by cervical spondylosis: a study protocol of a multicentre randomized controlled trial. Trials. 2012;13:107.

2. Tetreault LA, Karpova A, Fehlings MG. Predictors of outcome in patients with degenerative cervical spondylotic myelopathy undergoing surgical treatment: results of a systematic review. Eur Spine J. 2015;2:236-51.

3. Kumar VG, Rea GL, Mervis LJ, McGregor JM. Cervical spondylotic myelopathy: functional and radiographic long-term 
outcome after laminectomy and posterior fusion. Neurosurgery. 1999;44:771-8

4. Nicotra A, King NK, Catley M, Mendoza N, McGregor AH, Strutton PH. Evaluation of corticospinal excitability in cervical myelopathy, before and after surgery, with transcranial magnetic stimulation: a pilot study. Eur Spine J. 2013;22:189-96.

5. Kimura A, Seichi A, Endo T, Norimatsu Y, Inoue H, Higashi T, et al. Tally counter test as a simple and objective assessment of cervical myelopathy. Eur Spine J. 2013;22:183-8.

6. Hosono N, Sakaura H, Mukai Y, Kaito T, Makino T, Yoshikawa H. A simple performance test for quantifying the severity of cervical myelopathy. J Bone Jt Surg Br. 2008;90:1210-3.

7. Ono K, Ebara S, Fuji T, Yonenobu K, Fujiwara K, Yamashita K. Myelopathy hand. New clinical signs of cervical cord damage. J Bone Jt Surg Br. 1987;69:215-9.

8. Kato S, Oshima Y, Oka H, Chikuda H, Takeshita Y, Miyoshi K, et al. Comparison of the Japanese Orthopaedic Association (JOA) score and modified JOA (mJOA) score for the assessment of cervical myelopathy: a multicenter observational study. PLoS ONE. 2015. https://doi.org/10.1371/journal.pone.0123022.

9. Akutagawa T, Tani T, Kida K, Tadokoro N, Enoki H, Nagano Y, et al. A new method for characterizing hand dysfunction in cervical spondylotic myelopathy: a preliminary study. Spinal Cord. 2016;54:221-5

10. Nowak DA, Hermsdörfer J. Grip force behavior during object manipulation in neurological disorders: toward an objective evaluation of manual performance deficits. Mov Disord. 2005;20:11-25.

11. Lang CE, Schieber MH. Stroke. In: Nowak DA, Hermsdörfer J, editors. Sensorimotor control of grasping. 1st ed. Cambridge: Cambridge University Press; 2009. p. 296-310.

12. Blennerhassett JM, Carey LM, Matyas TA. Grip force regulation during pinch grip lifts under somatosensory guidance: comparison between people with stroke and healthy controls. Arch Phys Med Rehabil. 2006;87:418-29.

13. McDonnell MN, Hillier SL, Ridding MC, Miles TS. Impairments in precision grip correlate with functional measures in adult hemiplegia. Clin Neurophysiol. 2006;117:1474-80.

14. Westling G, Johansson RS. Factors influencing the force control during precision grip. Exp Brain Res. 1984;53:277-84.

15. Fujiwara H, Makino T, Yonenobu K, Honda H, Kaito T. Correlations and time-dependent changes of upper arm performance tests, the Japanese Orthopaedic Association score, and a newly developed patient-based outcome measure: the Japanese
Orthopaedic Cervical Myelopathy Questionnaire. Bone Jt J. 2016;98-B:990-6.

16. Shindo K, Oba H, Hara J, Ito M, Hotta F, Liu M. Psychometric properties of the simple test for evaluating hand function in patients with stroke. Brain Inj. 2015;29:772-6.

17. Seo NJ, Rymer WZ, Kamper DG. Altered digit force direction during pinch grip following stroke. Exp Brain Res. 2010;202:891-901.

18. Lee B, Noguchi N, Kakiage D, Yamazaki T. Kinematic characteristics of grip force in patients with cervical spondylosis. Phys Ther Rehabil Sci. 2015;4:61-5.

19. Chiles BW III, Leonard MA, Choudhri HF, Cooper PR. Cervical spondylotic myelopathy: patterns of neurological deficit and recovery after anterior cervical decompression. Neurosurgery. 1999;44:762-9.

20. Crandall PH, Batzdorf U. Cervical spondylotic myelopathy. J Neurosurg. 1966;25:57-66.

21. Marzke MW. Upper-limb evolution and development. J Bone Jt Surg Am. 2009;91:26-30.

22. Sasaki S, Isa T, Pettersson LG, Alstermark B, Naito K, Yoshimura $\mathrm{K}$, et al. Dexterous finger movements in primate without monosynaptic corticomotoneuronal excitation. J Neurophysiol. 2004;92:3142-7.

23. Woolsey CN, Erickson TC, Gilson WE. Localization in somatic sensory and motor areas of human cerebral cortex as determined by direct recording of evoked potentials and electrical stimulation. J Neurosurg. 1979;51:476-506.

24. Funaba M, Kanchiku T, Imajo Y, Suzuki H, Yoshida Y, Nishida $\mathrm{N}$, et al. Characteristics of C6-7 myelopathy: assessment of clinical symptoms and electrophysiological findings. Spinal Cord. 2016;54:798-803.

25. Bunday KL, Tazoe T, Rothwell JC, Perez MA. Subcortical control of precision grip after human spinal cord injury. J Neurosci. 2014;34:7341-50.

26. Johansson RS, Westling G. Roles of glabrous skin receptors and sensorimotor memory in automatic control of precision grip when lifting rougher or more slippery objects. Exp Brain Res. 1984;56:550-64.

27. Singh A, Crockard HA. Quantitative assessment of cervical spondylotic myelopathy by a simple walking test. Lancet. 1999;31:370-3.

28. Kalsi-Ryan S, Beaton D, Curt A, Duff S, Popovic MR, Rudhe C, et al. The graded redefined assessment of strength sensibility and prehension: reliability and validity. J Neurotrauma. 2012;29:905-14. 